\title{
Lack of causal relationship between inducibility/ severity of adjuvant arthritis in the rat and disease associated changes in production of nitric oxide by macrophages
}

\author{
Zdeněk Zidek, Daniela Franková, Berta Otová
}

\begin{abstract}
Objective-To investigate the formation of nitric oxide (NO) by peritoneal macrophages in three inbred strains of rats differing both in their susceptibility to the induction of adjuvant arthritis (AA) and in the severity of the disease.

Methods-AA was induced by intraplantar injection of Mycobacterium tuberculosis (M.tb) in paraffin oil. Isolated peritoneal macrophages were cultured for 24 hours and supernatants were assayed for nitrite using Griess reagent.

Results-All rats of the LEW and $B N$ strains became diseased, but the F344 strain included both responders and nonresponders. No significant interstrain differences were observed in the generation of NO by macrophages from control animals. Nitrite concentrations were remarkably enhanced in all M.tb treated animals, regardless of the absence or presence of $A A$, and did not parallel its severity. Altered production of NO by macrophages from adjuvant treated rats was normalised in vitro in the presence of lipopolysaccharide.

Conclusions-Our findings suggest that the activity of constitutive or inducible NO synthase in peritoneal macrophages cannot be regarded as a determinant of genetically controlled disease inducibility and severity. Secretion of latent forms of certain NO downregulatory factors during development of AA may be implicated.
\end{abstract}

Institute of

Pharmacology,

Academy of Sciences

of Czech Republic,

Prague, Czech

Republic

Z Zidek

D Franková

Department of

Biology, 1st Faculty of

Medicine, Charles

University, Prague,

Czech Republic

B Otová

Correspondence to:

Z Zídek,

Institute of Pharmacology, Laboratory of Immunology Academy of Sciences of Czech Republic, Videňskà 1083, 14220 Prague 4, Czech Republic.

Accepted for publication 11 November 1994
(Ann Rheum Dis 1995; 54: 325-327)

Although exact understanding of the aetiology and pathogenetic aspects of adjuvant induced arthritis (AA) in the rat remains to be established, it is generally regarded as a $T$ cell dependent chronic inflammatory disease of autoimmune origin. ${ }^{12}$ Activated $T$ lymphocytes are a well recognised major source of a number of cytokines, ${ }^{3}$ some of which, either per se or in co-operation with other macrophage derived factors (monokines), stimulate biosynthesis of nitric oxide (NO). ${ }^{4}$ Cytokines such as interferon gamma (IFN $\gamma$ ), tumour necrosis factor $\alpha$ (TNF $\alpha$ ), interleukin (IL)-1, IL-2, or IL-6, have been shown to be implicated in the development of immune arthritis. ${ }^{5-8}$ It is not surprising, therefore, that changes in NO production have been found to be associated with arthritic immunopathies, ${ }^{9}$ though the actual relationship of NO to disease pathogenesis is not well defined.

The aim of this study was to investigate changes in the capability of macrophages to generate NO during development of AA in rats, and to examine if these changes are related to the inducibility or severity of the disease.

\section{Materials and methods}

ANIMALS

Female rats of the inbred strains Lewis (LEW), Brown Norway (BN) and Fischer (F344) (weights $150-160 \mathrm{~g}$ ) were obtained from Charles River Wiga, Germany.

INDUCTION OF ARTHRITIS AND ITS EVALUATION Adjuvant arthritis was induced by intraplantar injection of $0.5 \mathrm{mg}$ Mycobacterium tuberculosis (strain H37RA, Difco) (M.tb) in $0.1 \mathrm{ml}$ of liquid paraffin. Control animals were injected with saline. Swelling of the uninjected paw was evaluated using a plethysmometer (Ugo Basile 7150).

CELL CULTURES

Peritoneal cells were collected 5, 11, and 21 days after the $M . t b$ injection, resuspended in RPMI-1640 medium, and placed in 96 well microplates $\left(2.5 \times 10^{5}\right.$ cells/well $)$. After a standard adherence procedure, the medium was replaced with complete RPMI-1640 medium containing $10 \%$ heat inactivated fetal bovine serum (Flow ICN), $50 \mu \mathrm{g} / \mathrm{ml}$ gentamicin, $2 \mathrm{mmol} / \mathrm{l}$ L-glutamine, and $5 \times 10^{-5}$ $\mathrm{mol} / \mathrm{l}$ 2-mercaptoethanol (Sigma). The cultures were maintained for 24 hours at $37^{\circ} \mathrm{C}$, in $5 \%$ carbon dioxide, in a humidified Heraeus incubator. Samples from individual animals were run in triplicate. In a parallel series, cells were incubated in the presence of lipopolysaccharide (LPS) (Salmonella typhimurium, Sigma), $5 \mu \mathrm{g} / \mathrm{ml}$. The final volume per well was always $100 \mu \mathrm{l}$.

\section{NITRITE ASSAY}

The amount of nitrite (expressed as $\mathrm{nmol} / 10^{6}$ cells) in the macrophage supernatants, taken as 
Swelling of the uninjected paw in adjuvant treated rats during the observation period of 21 days

\begin{tabular}{|c|c|c|c|c|c|}
\hline \multirow[t]{2}{*}{ Strain } & \multirow[t]{2}{*}{ Group } & \multirow[t]{2}{*}{$n$} & \multicolumn{3}{|c|}{ Volume of swelling $(\mathrm{ml})$} \\
\hline & & & Day 11 & Day 15 & Day 21 \\
\hline \multirow[t]{2}{*}{ LEW } & Control & 6 & $0.01(0.02)$ & $0.02(0.01)$ & $0.01(0.03)$ \\
\hline & M. $t b$ treated & 6 & $0.40(0.11)^{\star}$ & $1.02(0 \cdot 13)^{\star \star \star}$ & $1.23(0.08)^{\star \star \star}$ \\
\hline \multirow[t]{2}{*}{$\mathrm{BN}$} & Control & 6 & $0.01(0.01)$ & $0.02(0.03)$ & $0.03(0.05)$ \\
\hline & M. $t b$ treated & 6 & $0.04(0.03)$ & $0.08(0.04)$ & $0.63(0 \cdot 12)^{\star \star}$ \\
\hline \multirow[t]{3}{*}{ F344 } & Control & 6 & $-0.02(0.02)$ & $0.03(0.03)$ & $0.03(0.02)$ \\
\hline & $\begin{array}{l}\text { M. } t b \text { treated } \\
\quad(\text { responders })\end{array}$ & 6 & $0.08(0.03)^{\star}$ & $0 \cdot 15(0.04)^{\star}$ & $0 \cdot 46(0 \cdot 12)^{\star}$ \\
\hline & $\begin{array}{l}\text { M.tb treated } \\
\text { (non-responders) }\end{array}$ & 4 & $-0.03(0.04)$ & $0.03(0 \cdot 02)$ & $0.05(0.03)$ \\
\hline
\end{tabular}

Values are mean(SEM). M.tb $=$ Mycobacterium tuberculosis.

Significantly different from zero increase: ${ }^{\star} \mathrm{p}<0.05 ;{ }^{\star \star} \mathrm{p}<0.01 ;{ }^{\star \star \star} \mathrm{p}<0.001$

a measure of NO production, was determined using a Griess reagent and read at $540 \mathrm{~nm}$.

STATISTICAL ANALYSIS

Analysis of variance and Student's $t$ test were used to establish the significance of differences between the group means.

\section{Results}

DEVELOPMENT OF ADJUVANT ARTHRITIS

As shown in the table, AA (swelling) developed in all animals of the LEW $(n=6)$ and $\mathrm{BN}$ $(n=6)$ strains, whereas only four of 10 treated F344 rats became arthritic. The onset of AA was delayed in BN and F344 strains compared with LEW rats. The most severe response was observed in LEW animals.

NITRITE FORMATION AND IN VITRO EFFECT OF LIPOPOLYSACCHARIDE

Figure 1 shows NO formation on day 21 in control animals, AA responders and nonresponders, in the presence or absence of LPS.

No significant strain differences were found in NO production by macrophages from

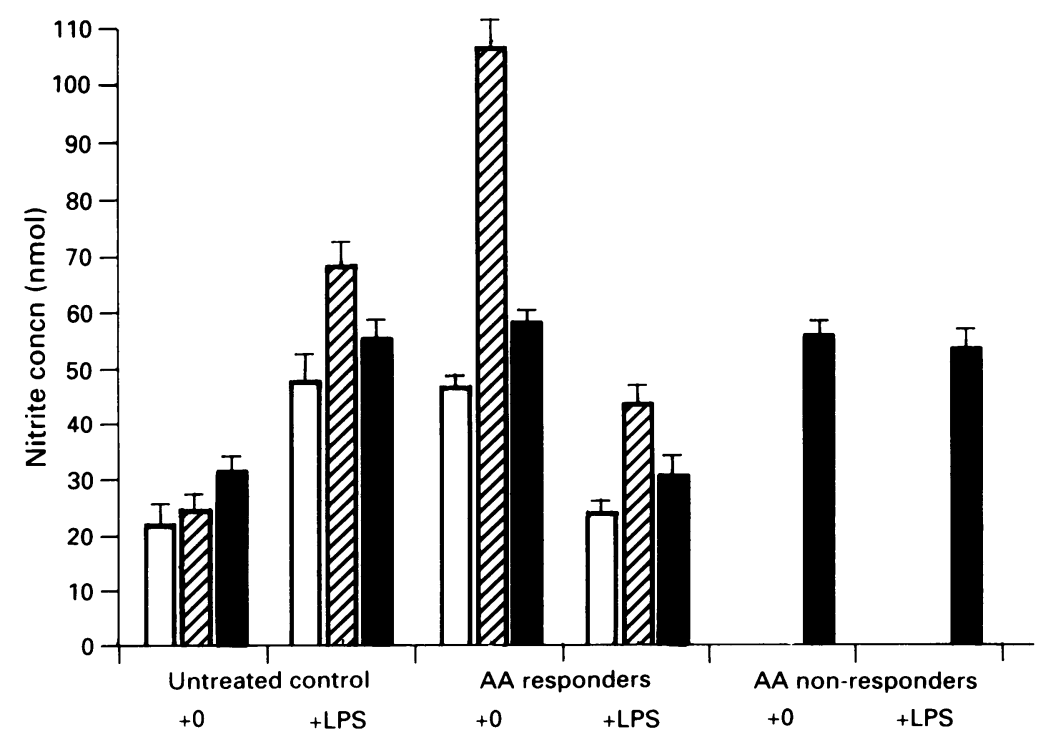

Figure 1 Concentration of nitrite ( $\mathrm{nmol} / 10^{6}$ cells) in supernatants of peritoneal macrophages collected from $L E W(\square), B N(\bigotimes)$ and $F 344$ ( $\square$ ) rats on day 21 after intraplantar injection of M. tuberculosis. Macrophages were cultured either alone or in the presence of lipopolysaccharide (LPS) $5 \mu \mathrm{g} / \mathrm{ml}$. Values are means and SEM for six animals (four animals for F344 non-responders). No non-responders occurred in the LEW and BN strain. $A A=$ adjuvant arthritis. control animals not treated with M.tb, but production was significantly enhanced $(p<0.001)$ after in vitro coincubation of cells with LPS.

Macrophages from adjuvant treated rats produced nitrites in markedly increased amounts $(p<0.001)$ which differed between the genotypes. However, when the cells were cultured in the presence of LPS, their augmented ability to produce NO was reduced to that of LPS untreated macrophages from control animals not injected with $M$.tb, except for those of strain $\mathrm{BN}$, in which the return to normal values was incomplete.

The disease free F344 animals (nonresponders) showed an increased production of NO which was indistinguishable from that detected in their F344 diseased (responders) counterparts. In this group, however, the increase was not affected by the in vitro presence of LPS.

A trypan blue exclusion test showed that $87-93 \%$ of cells from both control and arthritic animals remained viable at the end of the 24 hour period of culture, regardless of the presence or absence of LPS.

DYNAMICS OF CHANGES IN PRODUCTION OF NO Significantly increased production of NO was observed throughout the study period (days 5 , 11, and 21) (fig 2). NO production remained increased in the in vitro presence of LPS on day $5(p>0 \cdot 20)$, but had decreased significantly on days 11 and $21(\mathrm{p}<0.001)$.

\section{Discussion}

These results demonstrate that peritoneal macrophages from rats injected with mycobacterial adjuvant produced enhanced amounts of $\mathrm{NO}$ and that this effect preceded the onset of secondary arthritic lesions. These findings are compatible with those of Ialenti $e t$ $a l^{10}$ who showed that the severity of arthritis in Lewis rats was paralleled by pharmacologically manipulated production of $\mathrm{NO}$ by peritoneal macrophages. Others have reported a reduction of arthritic swelling in rats by inhibition of $\mathrm{L}$-arginine dependent formation of NO. ${ }^{11}{ }^{12}$ Considered alone, these data might suggest that increased production of NO is one of the pathogenetic mechanisms of AA; however, our comparative genetic study does not support such an unambiguous conclusion. We found also that macrophages from $M . t b$ treated (but disease-free) rats of the F344 strain produced similarly enhanced concentrations of NO. Furthermore, we found no association between severity of the AA and the magnitude of the increase in NO production; rather, the increase in production of $\mathrm{NO}$ was greater in $\mathrm{BN}$ rats which showed a very mild arthritic response, and least in LEW rats with the most severe AA. Moreover, changes in NO production in F344 mild responders were similar to those found in LEW rats. In addition, no quantitative differences in formation of $\mathrm{NO}$ existed between AA susceptible and $\mathrm{AA}$ resistant $\mathrm{F} 344$ rats. 


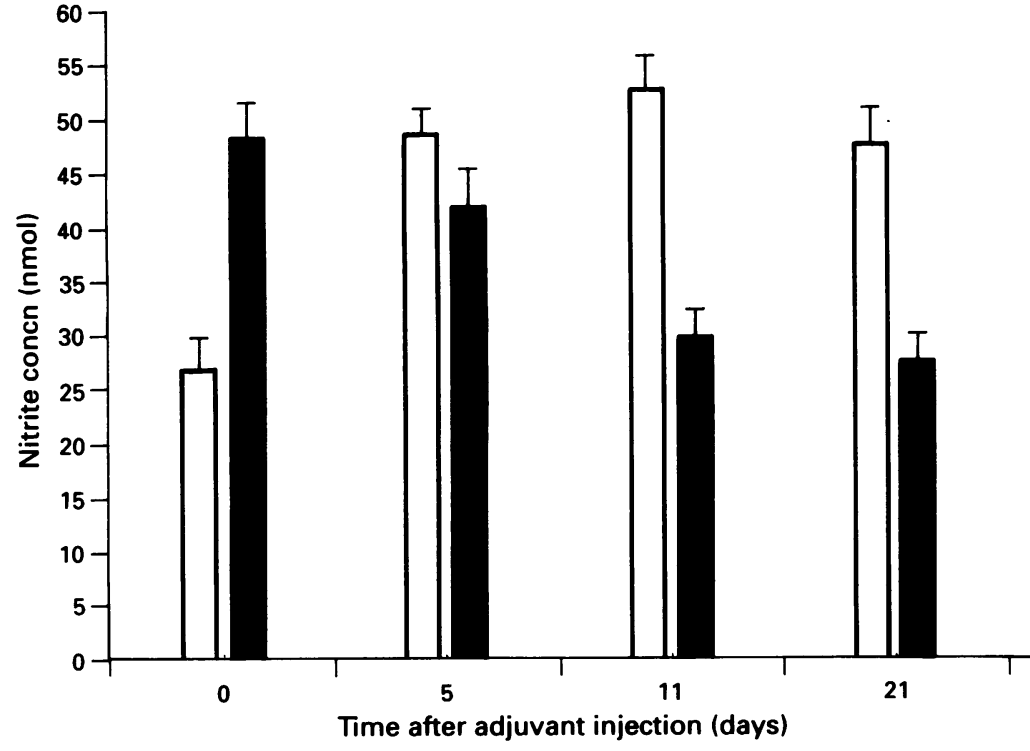

Figure 2 Production of nitrite ( $\mathrm{Nmol} 10^{6}$ cells) by peritoneal macrophages from $L E W$ rats injected with mycobacterial adjuvant. Macrophages were cultured either alone ( $\square$ ) or in the presence of lipopolysaccharide $5 \mu \mathrm{g} / \mathrm{ml}$ ( $\mathbf{(})$. Values are means and SEM for six animals; those at time 0 represent untreated controls.

Overall, these findings show that increased production of NO by peritoneal macrophages from adjuvant treated rats is not a correlate of genetically controlled disease inducibility, nor is it a biochemical marker of disease severity. It remains to be clarified, however, whether systemic changes in NO production, assessed here in peritoneal macrophages, reflect the situation at the site of inflammation. It cannot be excluded that local formation of NO is essential for the manifestation of arthritis. In any case, our findings support the view that NO should be regarded as a modifier rather than a direct mediator of the disease. Further pharmacogenetically designed studies to monitor constitutive and inducible NO synthase activity directly in the microenvironment of target joints and adjacent tissues will be undertaken to elucidate this problem.

Our results also show that macrophages from arthritic rats lose their enhanced capacity to generate NO when they are cultured in vitro with LPS. This effect was only expressed in AA responders, and was fully manifested at the time of onset of the swelling. It is difficult to explain the mechanism and biological significance of this phenomenon at present. Further studies are required to ascertain if 'arthritic macrophages' can secrete latent forms of downregulatory factors of $\mathrm{NO}$ synthesis, which may be rendered biologically active when exposed to LPS. Such biological properties are known to be inherent to transforming growth factor $\beta-1^{13} 14$ which, interestingly, has been shown to be implicated in arthritic disease. ${ }^{6}$

This work was supported by Grant No 307/94/0964 awarded by the Czech Republic.

1 Kayashime K, Koga T, Onoue K. Role of T lymphocytes in adjuvant arthritis. II. Different subpopulations of T lymphocytes functioning in the development of the disease. F Immunol 1978; 120: 1127-31.

2 Cohen I R. Autoimmunity to chaperonins in the pathogenesis of arthritis and diabetes. Ann Rev Pharmacol 1991; 9: 567-89.

3 Mosmann T R, Schumacher J H, Street N F, et al. Diversity of cytokine synthesis and function of mouse $\mathrm{CD}^{+} \mathrm{T}$ cells. Immunol Rev 1991; 123: 209-29.

4 Deng W, Thiel B. Tannenbaum C S, Hamilton T A, Stuehr D J. Synergistic cooperation between T cell lymphokines for induction of the nitric oxide synthase gene in murin peritoneal macrophages. F Immunol 1993; 151: 322-9.

5 Cooper S M, Sriram S, Ranges G E. Suppression of murin collagen-induced arthritis with monoclonal anti-Ia antibodies and augmentation with IFN- $\gamma$. F Immunol 1988; 141: $1958-62$.

6 Thorbecke G J, Shah R, Leu C H, Kuruvilla A P, Hardison A M, Palladino M A. Involvement of endogenous TNF- $\alpha$ and TGF- $\beta$ during induction of collagen type II arthritis in mice. Proc Natl Acad Sci USA 1992; 89: 7375-9.

7 Maury C P J. Interleukin 1 and the pathogenesis of inflammatory disease. Acta Med Scand 1986; 220: 291-4.

8 Theisen-Popp P, Pape H, Muller-Peddinghous R. Interleukin-6 (IL-6) in adjuvant arthritis of rats and its pharmacological modulation. $f$ Immunopharmacol 1992; pharmacological

9 Farrell A J, Blake D R, Palmer R M J, Moncada S. Increased concentrations of nitrite in synovial fluid and serum samples suggest increased nitric oxide synthesis in rheumatic diseases. Ann Rheum Dis 1992; 51: 1219-22.

10 Ialenti A, Moncada S, Di Rosa M. Modulation of adjuvant arthritis by endogenous nitric oxide. Br f Pharmacol 1993 110: 701-6.

11 McCartney-Francis N, Allen J B, Mizel D E, et al. Suppression of arthritis by an inhibitor of nitric oxide synthase. F Exp Med 1993; 178: 749-54

12 Oyanagui $Y$. Nitric oxide and superoxide radical are involved in both initiation and development of adjuvant arthritis in rats. Life Sci 1994; 54: 285-9.

13 Gilbert R S, Herschma H R Transforming growth factor beta differentially modulates the inducible nitric oxide synthase gene in distinct cell types. Biochem Biophys Res Commun 1993; 195: 380-4.

14 Corradin S B, Buchmuller-Rouiller Y, Smith J, Suardet L Mauel J. Transforming growth factor beta 1 regulation of macrophage activation depends on the triggering stimulus. F Leukoc Biol 1993; 54: 423-9. 